\title{
New Approach to the Generalized Poincare Conjecture
}

\author{
Alexander A. Ermolitski \\ IIT-BSUIR, Minsk, Belarus \\ Email: ermolitski@mail.by \\ Received April 18, 2013; revised May 18, 2013; accepted May 28, 2013
}

Copyright (C) 2013 Alexander A. Ermolitski. This is an open access article distributed under the Creative Commons Attribution License, which permits unrestricted use, distribution, and reproduction in any medium, provided the original work is properly cited.

\begin{abstract}
Using our proof of the Poincare conjecture in dimension three and the method of mathematical induction a short and transparent proof of the generalized Poincare conjecture (the main theorem below) has been obtained. Main Theorem. Let $M^{n}$ be a n-dimensional, connected, simply connected, compact, closed, smooth manifold and there exists a smooth finite triangulation on $M^{n}$ which is coordinated with the smoothness structure of $M^{n}$. If $S^{n}$ is the $n$-dimensional sphere then the manifolds $M^{n}$ and $S^{n}$ are homemorphic.
\end{abstract}

Keywords: Compact Smooth Manifolds; Riemannian Metric; Smooth Triangulation; Homotopy-Equivalence; Algorithms

\section{Introduction}

We can fix some Riemannian metric $g$ on a manifold $M^{n}$ of dimension $n$ which defines the length of arc of a piecewise smooth curve and the continuous function $\rho(x ; y)$ of the distance between two points $x, y \in M^{n}$. The topology defined by the function of distance (metric) $\rho$ is the same as the topology of the manifold $M^{n}$ [1].

In Section 1, using a smooth triangulation considered in the main theorem and a Riemannian metric we construct an algorithm of extension of coordinate neighborhood. With the help of this algorithm we get that every compact, connected, closed manifold $M^{n}$ of dimension $n$ having the triangulation above can be represented as a union of a $n$-dimensional cell $C^{n}$ and a connected union $K^{n-1}$ of some finite number of simplexes of the triangulation having dimension less or equal $(n-1)$. A sufficiently small closed neighborhood of $K^{n-1}$ is called a geometric black hole [2]. Simplexes with boundaries can be retracted i.e. a decomposition $M^{n}=\tilde{C}^{n} \cup \tilde{K}^{n-1}$ can be obtained where $\tilde{K}^{n-1}$ contains less simplexes than $\tilde{K}^{n-1}$ does.

In Section 2, we consider the proof of the main theorem consisting of the realization of several algorithms. Using the method of mathematical induction and the algorithms we retract all the simplexes from $\tilde{K}^{n-1}$ to a point $x_{0}$, therefore a decomposition $M^{n}=C^{n} \cup\left\{x_{0}\right\}$ is obtained and $M^{n}$ is homeomorphic to the sphere $S^{n}$.

\section{On Algorithm of Extension of Coordinate Neighborhood}

1) Let $M^{n}$ be a connected, compact, closed and smooth manifold of dimension $n$ and $C^{n}$ be a cell (coordinate neighborhood) on $M^{n}$. A standard simplex $\Delta^{n}$ of dimension $n$ is the set of points $x=\left(x_{1}, x_{2}, \cdots, x_{n}\right) \in \boldsymbol{R}^{n}$ defined by conditions

$$
0 \leq x_{i} \leq 1, i=1, \cdots n, x_{1}+x_{2}+\cdots+x_{n} \leq 1 .
$$

We consider the interval of a straight line connected the center of some face of $\Delta^{n}$ and the vertex which is opposite to this face. It is clear that the center of $\Delta^{n}$ belongs to the interval. We can decompose $\Delta^{n}$ as a set of intervals which are parallel to that mentioned above. If the center of $\Delta^{n}$ is connected by intervals with points of some face of $\Delta^{n}$ then a subsimplex of $\Delta^{n}$ is obtained. All the faces of $\Delta^{n}$ considered, $\Delta^{n}$ is seen as a set of all such subsimplexes. Let $U\left(D^{n}\right)$ be some open neighborhood of $\Delta^{n}$ in $\boldsymbol{R}^{n}$. A diffeomorphism

$\varphi: U\left(\Delta^{n}\right) \rightarrow M^{n}\left(\delta^{n}=\varphi\left(\Delta^{n}\right)\right)$ is called a singular $n$ simplex on the manifold $M^{n}$. Faces, edges, the center, vertexes of the simplex $\delta^{n}$ are defined as the images of those of $\Delta^{n}$ with respect to $\varphi$.

The manifold $M^{n}$ is triangulable [3]. It means that for any $l, 0 \leq l \leq n$ such a finite set $\Phi^{l}$ of diffeomorphisms $\varphi: \Delta^{l} \rightarrow M^{n}$ is defined that

a) $M^{n}$ is a disjunct union of images $\varphi\left(\operatorname{Int} \Delta^{l}\right), \varphi \in \Phi^{l}$; 
b) if $\varphi \in \Phi^{l}$ then $\varphi \circ \varepsilon_{i} \in \Phi^{l-1}$ for every $i$ where $\varepsilon_{i}: \Delta^{k-1} \rightarrow \Delta^{k}$ is the linear mapping transferring the vertexes $v_{0}, \cdots, v_{k-1}$ of the simplex $\Delta^{k-1}$ in the vertexes $v_{0}, \cdots, \hat{v}_{i}, \cdots, v_{k}$ of the simplex $\Delta^{k}$.

We suppose that there exists a smooth finite triangulation on $M^{n}$ which is coordinated with the smoothness structure of $M^{n}$ and fix the triangulation. Such triangulations exist for manifolds of dimension 2 or 3 .

2) Let $\delta_{0}^{n}$ be some simplex of the fixed triangulation of the manifold $M^{n}$. We paint the inner part $\operatorname{Int} \delta_{0}^{n}$ of the simplex $\delta_{0}^{n}$ white and the boundary $\partial \delta_{0}^{n}$ of $\delta_{0}^{n}$ black. There exist coordinates on $\operatorname{Int} \delta_{0}^{n}$ given by diffeomorphism $\varphi_{0}$. A subsimplex $\delta_{01}^{n-1} \subset \delta_{0}^{n}$ is defined by a black face $\delta_{01}^{n-1} \subset \delta_{0}^{n}$ and the center $c_{0}$ of $\delta_{0}^{n}$. We connect $c_{0}$ with the center $d_{0}$ of the face $\delta_{01}^{n-1}$ and decompose the subsimplex $\delta_{01}^{n}$ as a set of intervals which are parallel to the interval $c_{0} d_{0}$. The face $\delta_{01}^{n-1}$ is a face of some simplex $\delta_{1}^{n}$ that has not been painted. We draw an interval between $d_{0}$ and the vertex $v_{1}$ of the subsimplex $\delta_{1}^{n}$ which is opposite to the face $\delta_{01}^{n-1}$ then we decompose $\delta_{1}^{n}$ as a set of intervals which are parallel to the interval $d_{0} v_{1}$. The set $\delta_{01}^{n} \cup \delta_{1}^{n}$ is a union of such broken lines every one from which consists of two intervals where the endpoint of the first interval coincides with the beginning of the second interval (in the face $\delta_{01}^{n-1}$ ) the first interval belongs to $\delta_{01}^{n}$ and the second interval belongs to $\delta_{1}^{n}$. We construct a homeomorphism (extension) $\varphi_{01}^{1}: \operatorname{Int} \delta_{01}^{n} \rightarrow \operatorname{Int}\left(\delta_{01}^{n} \cup \delta_{1}^{n}\right)$. Let us consider a point $x \in \operatorname{Int} \delta_{01}^{n}$ and let $x$ belong to a broken line consisting of two intervals the first interval is of a length of $s_{1}$ and the second interval is of a length of $s_{2}$ and let $x$ be at a distance of $s$ from the beginning of the first interval. Then we suppose that $\varphi_{01}^{1}(x)$ belongs to the same broken line at a distance of $\frac{s_{1}+s_{2}}{s_{1}} \cdot s$ from the beginning of the first interval. It is clear that $\varphi_{01}^{1}$ is a homeomorphism giving coordinates on $\operatorname{Int}\left(\delta_{01}^{n} \cup \delta_{1}^{n}\right)$. We paint points of $\operatorname{Int}\left(\delta_{01}^{n} \cup \delta_{1}^{n}\right)$ white. Assuming the coordinates of points of white initial faces of subsimplex $\delta_{01}^{n}$ to be fixed we obtain correctly introduced coordinates on $\operatorname{Int}\left(\delta_{0}^{n} \cup \delta_{1}^{n}\right)$. The set $\sigma_{1}=\delta_{0}^{n} \cup \delta_{1}^{n}$ is called a canonical polyhedron. We paint faces of the boundary $\partial \sigma_{1}$ black.

We describe the contents of the successive step of the algorithm of extension of coordinate neighborhood. Let us have a canonical polyhedron $\sigma_{k-1}$ with white inner points (they have introduced white coordinates) and the black boundary $\partial \sigma_{k-1}$. We look for such an $n$-simplex in $\sigma_{k-1}$, let it be $\delta_{0}^{n}$ that has such a black face, let it be $\delta_{01}^{n-1}$ that is simultaneously a face of some $n$-simplex, let it be $\delta_{1}^{n}$, inner points of which are not painted. Then we apply the procedure described above to the pair $\delta_{0}^{n}, \delta_{1}^{n}$. As a result we have a polyhedron $\sigma_{k}$ with one simplex more than $\sigma_{k-1}$ has. Points of Int $\sigma_{k}$ are painted white and the boundary $\partial \sigma_{k}$ is painted black. The process is finished in the case when all the black faces of the last polyhedron border on the set of white points (the cell) from two sides.

After that all the points of the manifold $M^{n}$ are painted in black or white, otherwise we would have that $M^{n}=M_{0}^{n} \cup M_{1}^{n}$ (the points of $M_{0}^{n}$ would be painted and those of $M_{1}^{n}$ would be not) with $M_{0}^{n}$ and $M_{1}^{n}$ being unconnected, which would contradict of connectivity of $M^{n}$.

Thus, we have proved the following.

Theorem 1. Let $M^{n}$ be a connected, compact, closed, smooth manifold of dimension $n$. Then $M^{n}=C^{n} \cup K^{n-1}$, $C^{n} \cap K^{n-1}=\varnothing$, where $C^{n}$ is an n-dimensional cell and $K^{n-1}$ is a union of some finite number of $(n-1)$-simplexes of the triangulation.

3) We consider the initial simplex $\delta_{0}^{n}$ of the triangulation and its center $c_{0}$. Drawing intervals between the point $c_{0}$ and points of all the faces of $\delta_{0}^{n}$ we obtain a decomposition of $\delta_{0}^{n}$ as a set of the intervals. In 2) the homeomorphism $\psi: \operatorname{Int} \delta_{0}^{n} \rightarrow C^{n}$ was constructed and $\psi$ evidently maps every interval above on a piecewise smooth broken line $\gamma$ in $C^{n}$. We denote $\tilde{M}^{n}=M^{n} \backslash\left\{c_{0}\right\}$. $\tilde{M}^{n}$ is a connected and simply connected manifold if $M^{n}$ is that. Let $I=[0 ; 1]$, we define a homotopy $F: \tilde{M}^{n} \times I \rightarrow \tilde{M}^{n}:(x ; t) \mapsto y=F(x ; t)$ in the following way

a) $F(z ; t)=z$ for every point $z \in K^{n-1}$;

b) if a point $x$ belongs to the broken line $\gamma$ in $C^{n}$ and the distance between $x$ and its limit point $z \in K^{n-1}$ is $s(x)$ then $y=F(x ; t)$ is on the same broken line $\gamma$ at a distance of $(1-t) s(x)$ from the point $z$.

It is clear that $F(x ; 0)=x, F(x ; 1)=z$ and we have obtained the following.

Theorem 2. The spaces $\tilde{M}^{n}$ and $K^{n-1}$ are homotopyequivalent, in particular, the groups of singular homologies $H_{k}\left(\tilde{M}^{n}\right)$ and $H_{k}\left(K^{n-1}\right)$ are isomorphic for every $k$.

Corollary 2.1. The space $K^{n-1}$ is connected and if $M^{n}$ is simply connected then $K^{n-1}$ is simply connected too.

Remark 1. The white coordinates are extended from the simplex $\delta_{0}^{n}$ in the simplex $\delta_{1}^{n}$ through the face $\delta_{01}^{n-1}$ hence Int $\delta_{01}^{n-1}$ has also the white coordinates. On the other hand there exist two linear structures (intervals, the center etc.) on $\delta_{01}^{n}$ induced from $\delta_{0}^{n}$ and $\delta_{1}^{n} \mathrm{re-}$ spectively. Further, we set that the linear structure of $\delta_{01}^{n-1}$ is the structure induced from $\delta_{0}^{n}$.

Remark 2. In the process of getting of $C^{n}$ in 2) we can construct a maximal tree $L$ connecting by intervals all the centers of the n-simplexes of the triangulation via the centers of some white faces.

Conversely, if we have such a maximal tree $L$ connecting by intervals all the centers of the $n$-simplexes of 
the triangulation via the centers of some faces (any from two possible centers of a face can be chosen) then we can extend white coordinates from any simplex $\delta_{0}^{n}$ on the maximal cell $C^{n}$ as it was shown in 2). Thus, the maximal tree $L$ defines the maximal cell $C^{3}$ and white faces.

4) Definition 1. a) $A$ simplex $\delta^{k} \in K^{n-1}(k=\overline{1, n-1})$ is called free if it has at least one free face $\delta^{k-1}$ i.e. such a face that it is not a face of any other $k$-simplex from $K^{n-1}$.

b) An edge $\delta^{1}=x_{0} x_{1}$ is called semi-isolated if it is not an edge of any simplex from $K^{n-1}$. A semi-isolated edge $\delta^{1}$ is called isolated if it is free.

Let us have a free simplex $\delta^{k} \in K^{n-1}$ with some free face $\delta^{k-1}$. We consider such a polyhedron $\sigma$ that $\sigma$ is the set of all n-simplexes having common point with $\delta^{k-1}$.

Proposition 3. We can redistribute coordinates of white points of the polyhedron $\delta$ (retract the free simplex $\left.\delta^{k}\right)$ i.e. construct the corresponding mapping $\varphi_{\sigma}$ in such a way that the following conditions are fulfilled:

a) all the points of Int $\sigma$ are painted white i.e. have new white coordinates,

b) white coordinates of points of boundary faces of the polyhedron $\sigma$ are not changed.

Proof. a) We consider the unit disk $D^{2}$ having the center in the origin $O=y_{0}$ of the coordinate system $O x_{1} x_{2}$ of $\mathbf{R}^{2}$ and the radius $y_{0} y_{1}$.

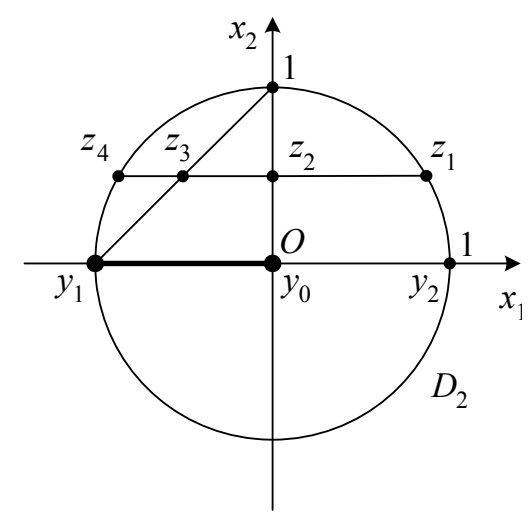

We define a mapping $\psi: D^{2} \rightarrow D^{2}$ by the following way:

- $\psi\left(y_{0} y_{2}\right)=y_{1} y_{2}, \psi\left(y_{0}\right)=y_{1}, \psi\left(y_{2}\right)=y_{2}$;

- for any chord $z_{1} z_{4}$ which is parallel to $y_{0} y_{1}$ $\psi\left(z_{1} z_{2}\right)=z_{1} z_{3}, \quad \psi\left(z_{2}\right)=z_{3}, \quad \psi\left(z_{1}\right)=z_{1}, \quad \psi\left(z_{2} z_{4}\right)=$ $z_{3} z_{4}, \psi\left(z_{4}\right)=z_{4}$.

It is clear that $\psi$ maps $\operatorname{Int} D^{2} \backslash y_{0} y_{1}$ onto $\operatorname{Int} D^{2}$ and $\psi=i d$ on the boundary circle of $D^{2}$.

b) We consider the unit disk $D^{k-1}: x_{1}^{2}+x_{2}^{2}+\cdots+x_{k-1}^{2}$ $\leq 1$ having the center in the origin $O$ of the coordinate system $O x_{1} x_{2} \cdots x_{k-1}$ and the semidisk $S D^{k-2}: x_{k-1}=0$, $x_{k-2} \leq 0, x_{1}^{2}+x_{2}^{2}+\cdots+x_{k-2}^{2} \leq 1$. By inductive hypothesis we assume that such a mapping $\psi: D^{k-1} \rightarrow D^{k-1}$ has been constructed that $\psi$ maps $\operatorname{Int} D^{k-1} \backslash S D^{k-2}$ onto $\operatorname{Int} D^{k-1}$ and $\psi=i d$ on the boundary of $D^{k-1}$.

Further, we consider the unit disk $D^{k}: x_{1}^{2}+x_{2}^{2}+\cdots$ $+x_{k}^{2} \leq 1$ in the coordinate system $O x_{1} x_{2} \cdots x_{k}$, the semidisk $S D^{k-1}: x_{k}=0, x_{k-1} \leq 0, \quad x_{1}^{2}+x_{2}^{2}+\cdots+x_{k-1}^{2} \leq 1$ and the family of disks $D_{t}^{k-1}: x_{1}^{2}+x_{2}^{2}+\cdots+x_{k}^{2} \leq 1$, $x_{k-2}=t, t \in[-1 ; 1]$. We denote $S D_{t}^{k-2}=D_{t}^{k-1} \cap S D^{k-1}$. By inductive hypothesis there exists such the family of mappings $\psi_{t}: D_{t}^{k-1} \rightarrow D_{t}^{k-1}(t \in[-1 ; 1])$ that every $\psi_{t}$ maps $\operatorname{Int} D_{t}^{k-1} \backslash S D_{t}^{k-2}$ onto $\operatorname{Int} D_{t}^{k-1}$ and $\psi_{t}=i d$ on the boundary of $D_{t}^{k-1}$. Union of all $\psi_{t}$ gives the mapping $\psi: D^{k} \rightarrow D^{k}, \psi$ maps $\operatorname{Int} D^{k} \backslash S D^{k-1}$ onto $\operatorname{Int} D^{k}$ and $\psi=i d$ on the boundary of $D^{k}$.

Thus, the mapping $\psi$ is constructed for any $n \in \mathbf{N}$ by the method of mathematical induction.

c) It is clear that there exists such a homeomorphism $\varphi: \sigma \rightarrow D^{n}$ that $\varphi(\partial \sigma)=\partial D^{n}$ and $\varphi\left(\delta^{k}\right) \subset S D^{n-1}$. We define the mapping $\varphi_{\sigma}=\varphi^{-1} \circ \psi \circ \varphi$ then $\varphi_{\sigma}: \operatorname{Int} \sigma \backslash \sigma^{k} \rightarrow \operatorname{Int} \sigma$ is a required homeomorphism introducing new white coordinates in $\operatorname{Int} \sigma$.

QED.

Remark 3. In is clear that the rebuilt complex $K^{n-1}$ is connected and simply connected because of a homotopy-equivalence.

5) We assume that in the process of painting free simplexes white by the Proposition 3 we get a representation $M^{n}=C^{n} \cup K^{1}, C^{n} \cap K^{1}=\varnothing$, where $K^{1}$ is the connected union of black edges of the triangulation. Since the process of painting free simplexes white does not influence simply connectivity of a space that has been obtained every step then $K^{1}$ is a tree if the complex $K^{n-1}$ is simply connected. Painting isolated edges of $K^{1}$ white by the Proposition 3 we have got unique black point $x_{0}$ as result. Thus, we obtain a representation $M^{n}=C^{n} \cup B^{n}\left(x_{0} ; \varepsilon\right)$, where $B^{n}\left(x_{0} ; \varepsilon\right)$ is an open geodesic ball with the center in $x_{0}$ and of a radius $\varepsilon$. The manifold $M^{n}$ is homeomorfic to the sphere $S^{n}$ by the following lemma 4 .

Lemma 4 [1]. If a topological manifold $M^{n}$ is a union of two n-dimensional cells then $M^{n}$ is homeomorfic to the sphere $S^{n}$.

\section{Proof of the Main Theorem}

The proof has a combinatorial nature and assumes the realization of a number of algorithms. We consider that step by step. The initial complex $K^{n-1}$ is assumed to be connected, simply connected and without free simplexes.

1) Proposition 5 (opening an input). Let $\delta_{1}^{n}$ be some n-simplex of the triangulation having a black face $\delta_{01}^{n-1} \in K^{n-1}$. Then $\operatorname{Int} \delta_{01}^{n-1}$ can be repainted white to get a new decomposition $M^{n}=C^{n} \cup K^{n-1}$, where $K^{n-1}$ is a new connected and simply connected complex.

Proof. The face $\delta_{01}^{n-1}$ is the common face of $n$-sim- 
plexes $\delta_{0}^{n}$ and $\delta_{1}^{n}$. We cansel the white painting of points of $\delta_{1}^{n}$ and paint the $n$-simplexe $\delta_{1}^{n}$ black. Repainting of $\delta_{1}^{n}$ black brings to a gap of the maximal tree $L$ (see the Remark 2) on $n$ subtrees $L_{1}, L_{2}, \cdots, L_{n}$ or less where the center of $\delta_{0}^{n}$ belongs to $L_{1}$. Further, we extend white coordinates from $\delta_{0}^{n}$ into $\delta_{1}^{n}$ through the face $\delta_{01}^{n-1}$ as it was shown in 2), 1 and connect the centers of $\delta_{0}^{n}, \delta_{01}^{n-1}, \delta_{1}^{n}$ by intervals. Those centers belong to the subtree $L_{1}$. Other faces of $\delta_{1}^{n}$ are black and they are simultaneously some faces of other $n$-simplexes.

We consider the following cases.

a) $L_{1}=L$ or we have no a gap. The black faces of $\delta_{1}^{n}$ remain black.

b) We have got $k$ subtrees $L_{1}, L_{2}, \cdots, L_{k}(k=\overline{2, n})$ where the subtrees $L_{2}, \cdots, L_{k}$ define cells called dead ends. We repaint the closures of the dead ends black. Further, we are looking for a black face of $\delta_{1}^{n}$ which is simultaneously a face of other $n$-simplex with the center from $L_{1}$. This face remains black. For every subtree $L_{i}(i=\overline{2, k})$ we consider a $n$-simplex with the center from $L_{i}$ that has a common black face $\delta_{1 i}^{n-1}$ with $\delta_{1}^{n}$. We extend white coordinates from $\delta_{1}^{n}$ through $\delta_{1 i}^{n-1}$ along the subtree $L_{i}$ as it was shown in 2), 1 and repaint inner points of this face and points of the corresponding dead end white. Further, we connect by intervals the centers of $\delta_{1 i}^{n-1}$ with the centers of $\delta_{1}^{n}$ and the other simplex connecting $L_{1}$ and $L_{i}$.

After repainting all the dead ends white we obtain a new maximal tree $L$ defining a new maximal cell $C^{3}$. Retracting all the free simplexes by the Proposition 3 a new rebuilt complex $K^{n-1}$ is obtained which is connected and simply connected because of homotopyequivalence.

QED.

Remark 4. A broken line has been obtained in the proof above which connects by intervals the centers of $\delta_{0}^{n}$, $\delta_{01}^{n-1}, \delta_{1}^{n}$. This broken line is a part of the subtree $L_{1}$ of the maximal tree L. Let n-simplexes $\delta_{0}^{n}$ and $\delta_{1}^{n}$ have a common face $\delta_{01}^{n-1}$ having the white inter part and $\operatorname{Int} \delta_{01}^{n-1}$ has no common points with the maximal tree $L$. Then we can connect the centers of $\delta_{0}^{n}, \delta_{01}^{n-1}$, $\delta_{1}^{n}$ by the broken line by the method considered in the proof above.

2) We assume the following inductive hypothesis:

The generalized Poincare conjecture (the main theorem) can be proved by the method considered in [4] for dimension $n-1$ i.e. the representation $M^{n-1}=C^{n-1} \cup\left\{x_{0}\right\}$ can be obtained by the algorithm from 2), 1 and by the Propositions 3, 4, 5.

It is obvious for $n-1=2$ (see 5), 1) It is proved for $n-1=3$ in [4].

We choose a small ball $B^{n}\left(x_{0}\right)$ with the center in a vertex $x_{0}$ which is diffeomorphic to a small ball in $\mathbf{R}^{n}$ and call a trace of $k$-simplex $\delta^{k}(k=\overline{1, n})$ with a vertex in $x_{0}$ its intersection $\bar{\delta}^{k-1}$ with the sphere $S^{n-1}\left(x_{0}\right)$ (smooth manifold) which is the boundary of $B^{n}\left(x_{0}\right)$. The sphere $S^{n-1}\left(x_{0}\right)$ is supposed to be transversal to all the $k$-simplexes $(k=\overline{1, n})$ with the vertex $x_{0}$. Such a sphere $S^{n-1}\left(x_{0}\right)$ exists because of the smoothness of the triangulation of $M^{n}[5,6]$. All other vertexes of the triangulation are supposed to be out of $B^{n}\left(x_{0}\right)$. The ball $B^{n}\left(x_{0}\right)$ can be chosen in such a vay that every edge with the endpoint $x_{0}$ has only one point of the intersection with $S^{n-1}\left(x_{0}\right)$ and every $k$-simplex $\delta^{k}$ with the vertex $x_{0}$ has only one connected component $\bar{\delta}^{k-1}$ of $\delta^{k} \cap S^{n-1}\left(x_{0}\right)$. Let $B s^{k}\left(x_{0}\right)$ be the set of black $k$-simplexes with $x_{0}$ as their vertex and $B s\left(x_{0}\right)=\bigcup_{k=1}^{n} B s^{k}\left(x_{0}\right)$. There exists one to one correspondence between the set of simplexes having a vertex (endpoint) $x_{0}$ and the set of their traces on $S^{n-1}\left(x_{0}\right)$ therefore all steps of the algorithm below bring to the corresponding steps on the sphere $S^{n-1}\left(x_{0}\right)$ and the converse is true. In particular, a process of the construction of a maximal tree $\bar{L}_{1}$ on the sphere $S^{n-1}\left(x_{0}\right)$ (see the Remark 2) brings to the construction of a tree $L_{1}$ connecting by intervals all the centers of the $n$-simplexes with $x_{0}$ as their vertex via the centers of some white their faces. Every such the face has $x_{0}$ as its vertex.

Proposition 6. The complex $K^{n-1}$ can be rebuilt in such a vay that $B s\left(x_{0}\right)$ contains only one 1-simplex $x_{0} x_{1}$.

Proof. We consider the smooth triangulation of $S^{n-1}\left(x_{0}\right)$ induced by all the simplexes with the vertex $x_{0}$ and apply to this triangulation the algorithm from 2), 1 taking any $(n-1)$-simplex $\bar{\delta}_{0}^{n-1}$ as initial one where $\bar{\delta}_{0}^{n-1}$ is the trace of $\delta_{0}^{n}$ with a vertex $x_{0}$. Let $\bar{\delta}_{0}^{n-1}$ be the trace on $S^{n-1}\left(x_{0}\right)$ of $\delta_{1}^{n}$ with a vertex $x_{0}$ where $\bar{\delta}_{1}^{n-1}$ has a common face with $\bar{\delta}_{0}^{n-1}$. We repaint $\delta_{1}^{n}$ black and apply to it the proposition 5 (the remark 4) obtaining the canonical polyhedron $\bar{\delta}_{0}^{n-1} \cup \bar{\delta}_{1}^{n-1}$ on $S^{n-1}\left(x_{0}\right)$. Further, we iterate the algorithm. Every step of the algorithm on $S^{n-1}\left(x_{0}\right)$ implies the transformation of $B s\left(x_{0}\right)$ and $K^{n-1}$ by the proposition 5 (the remark 4). The maximal tree $\bar{L}_{1}$ on $S^{n-1}\left(x_{0}\right)$ and the corresponding subtree $L_{1}$ have been constructed in the end. Further, free black simplexes on $S^{n-1}\left(x_{0}\right)$ and the corresponding free simplexes from $B s\left(x_{0}\right)$ can be annihilated by the propositions $3,4,5$. By the inductive hypothesis only one black point remains on $S^{n-1}\left(x_{0}\right)$ in the end. This point is the trace of an edge $x_{0} x_{1}$ which is isolated.

QED.

Remark 5. It is clear that if we paint black one inner vertex in the canonical polyhedron then we get two black 
points on $S^{n-1}\left(x_{0}\right)$ in the end of the algorithm.

3) We consider a small ball $B^{n}\left(x_{1}\right)$ with the center $x_{1}$ and the boundary $S^{n-1}\left(x_{1}\right)$ which is similar to $B^{n}\left(x_{0}\right)\left(S^{n-1}\left(x_{0}\right)\right)$. The centers of all the $n$-simplexes having $x_{0} x_{1}$ as their edge belong to the subtree $L_{1}$ and the union of all the traces of this $n$-simplexes on $S^{n-1}\left(x_{1}\right)$ forms the canonical polyhedron on $S^{n-1}\left(x_{1}\right)$ having one black inner vertex (the trace of isolated edge $x_{0} x_{1}$ ). We apply the Proposition 6 (the Remark 5) to the $S^{n-1}\left(x_{1}\right)$ and $B S\left(x_{1}\right)$. As a result $B S\left(x_{1}\right)$ consists of two semi-isolated edges $x_{0} x_{1}$ and $x_{1} x_{2}$.

Further, we iterate the process getting a broken line $x_{0} x_{1} \cdots x_{k}$ and for $i=\overline{1, k-1} B s\left(x_{\mathrm{i}}\right)$ consists of two black semi-isolated edges $x_{i-1} x_{i}$ and $x_{i} x_{i+1}$. We remark that the process of the annihilation of black simplexes in $B s\left(x_{\mathrm{i}}\right)$ cannot bring to an appearance of a black simplex having a generic point with $x_{j-1} x_{j}(j<i)$. Really, otherwise such a black simplex gives an opportunity to connect the endpoints $x_{i-1}$ and $x_{i}$ of the semi-isolated edge $x_{i-1} x_{i}$ by a black curve which is different from $x_{i-1} x_{i}$. As a result a black loop with the semi-isolated edge $x_{i-1} x_{i}$ as its part has been obtained and the loop is not contractible that is a contradiction to the simply con- nectivity of $K^{n-1}$.

The complex $K^{n-1}$ is connected therefore the broken line $x_{0} x_{1} \cdots x_{k}$ contains all the possible black vertexes from $K^{n-1}$ at some step of the algorithm i.e. we come to 5,1 .

By the method of mathematical induction the main theorem is true for every $n \in \mathbf{N}, n \neq 1$.

\section{REFERENCES}

[1] D. Gromoll, W. Klingenberg and W. Meyer, "Riemannsche Geometrie im Grossen," Springer, Berlin, 1968.

[2] A. A. Ermolitski, "On a Geometric Black Hole of a Compact Manifold," Intellectual Archive Journal, Vol. 1, No. 1, 2012, pp. 101-108.

[3] J. R. Munkres, "Elementary Differential Topology," Princeton University Press, Princeton, 1966.

[4] A. A. Ermolitski, "Three-Dimensional Compact Manifold and the Poincare Conjecture," Intellectual Archive Journal, Vol. 1, No. 4, 2012, pp. 51-62.

[5] D. B. Fuks and V. A. Rohlin, "Beginner's Course in Topology/Geometric Chapters," Nauka, Moscow, 1977.

[6] M. W. Hirsch, "Differential Topology," Springer, New York, Heigelberg, Berlin, 1976. 